\title{
Light Pseudoscalar Mesons: an Inverse Instantaneous Bethe-Salpeter Glimpse
}

\section{Wolfgang Lucha*}

Institute for High Energy Physics, Austrian Academy of Sciences, Nikolsdorfergasse 18, A-1050

Vienna, Austria

E-mail: Wolfgang. Lucha@oeaw.ac.at

\section{Franz F. Schöberl}

Faculty of Physics, University of Vienna, Boltzmanngasse 5, A-1090 Vienna, Austria

E-mail: franz.schoeberleunivie.ac.at

\begin{abstract}
Achieving self-consistent simultaneous interpretations of pions and kaons as bound states of quark and antiquark and as the (almost) massless boson states related, according to Goldstone's theorem, to the dynamical, and explicit, breakdown of the chiral symmetries of QCD still represents a major challenge. Applying inversion techniques to conveniently simplified versions of the homogeneous Bethe-Salpeter equation, governing bound states in quantum field theory, enables us to get along a straightforward route a qualitative idea of how the underlying effective interaction might look like.
\end{abstract}

The European Physical Society Conference on High Energy Physics 5-12 July 2017

Venice, Italy

\footnotetext{
* Speaker.
} 
For ground-state pseudoscalar mesons, their description as quark-antiquark bound states ought to reflect also their (near) masslessness, demanded by Goldstone's theorem for bosons related to the dynamical (and explicit) breaking of the chiral symmetries of quantum chromodynamics, the theory of the strong interactions. The underlying effective interactions enabling such combined picture can be elucidated or explored by inversion [1,2] of the Bethe-Salpeter formalism, suitably simplified by allowing for adequate instantaneous, hence three-dimensional, reductions [3,4]. From the latter, we may extract information [5-7] in form of configuration-space central potentials [8-10] $V(r), r \equiv|\mathbf{x}|$.

Strictly respecting Poincaré covariance, the homogeneous Bethe-Salpeter equation governing fermion-antifermion bound states is constructed from an integral kernel that subsumes the effective interactions responsible for the formation of the bound states and the propagators of the constituents of the bound states. Its solutions capture the distribution of the relative momenta of the constituents.

In view of evident difficulties to deduce the interaction kernel from quantum chromodynamics, we suggest to approach some of the information assumed to be encoded therein by applying more or less standard inversion procedures to sophisticated simplifications of the Bethe-Salpeter formalism, cast, for reasonably trivial dependence of kernel [11] and propagators [3] on the time components of all relevant momenta and flavour-, Fierz- and spherically symmetric interactions, into a bound-state problem for the Bethe-Salpeter solution's radial components. Only one of the latter, denoted by $\varphi_{2}$, matters for pseudoscalar mesons. Knowledge of solutions then sheds light on the basic interactions.

Invariance of a quantum field theory under gauge transformations entails identities which relate this theory's Green functions. In the chiral limit, one such identity connects [5,12] quark propagator and Bethe-Salpeter solution for a zero-mass pseudoscalar meson in its center-of-momentum frame.

Owing to Poincaré covariance, just two Lorentz-scalar functions characterize the propagator of a quark (of four-momentum $p$ ), its mass $M\left(p^{2}\right)$ and its wave-function renormalization $Z\left(p^{2}\right)$, which we take from a solution [13] of this quark propagator's Euclidean-space equation of motion (Fig. 1).
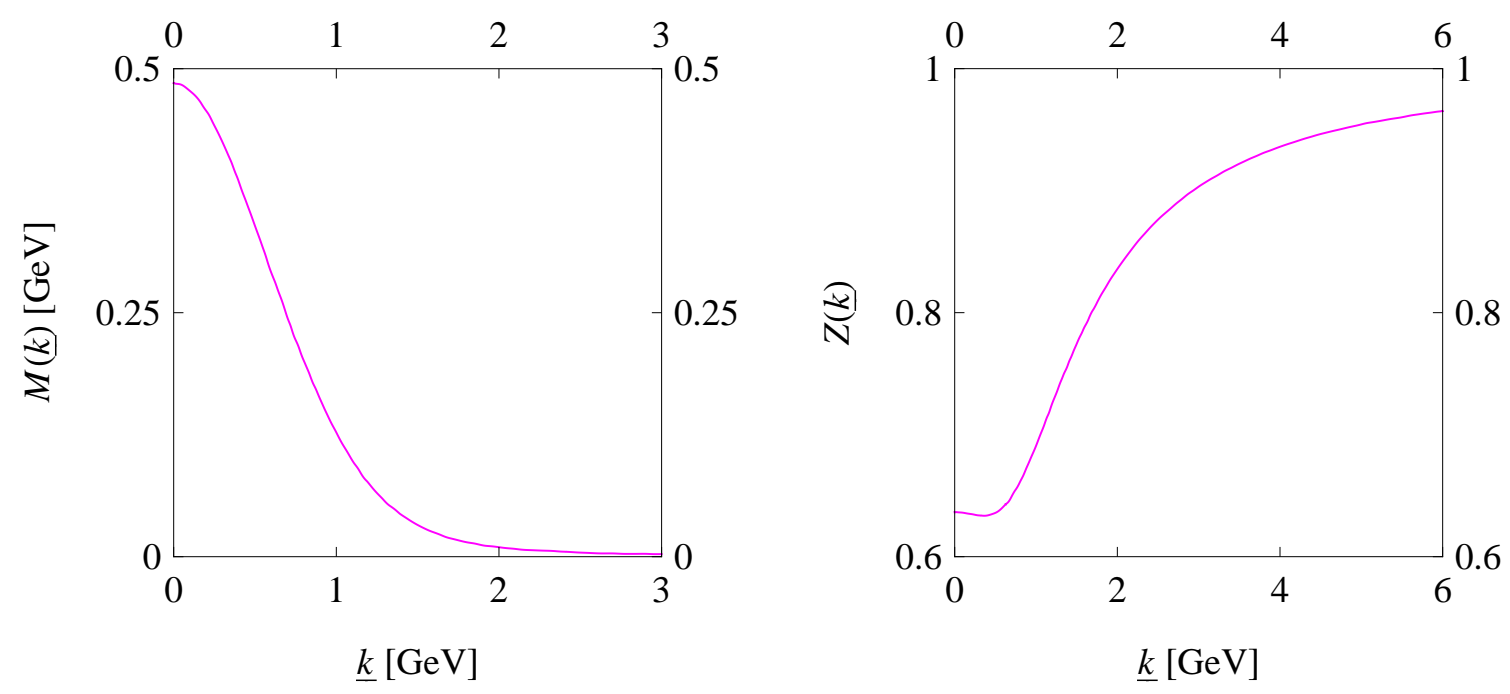

Figure 1: Euclidean quark propagator functions in chiral limit: $M(\underline{k})$ (left) and $Z(\underline{k})$ (right) vs. $\underline{k} \equiv \sqrt{\underline{k}^{2}}[14]$.

Inserting the propagator functions' behaviour plotted in Fig. 1 into the aforementioned identity opens the path [8] to the ground-state solution $\varphi_{2}(p)$, with radial variable $p \equiv|\mathbf{p}|$, and $\varphi_{2}(r)$ (Fig. 2). 

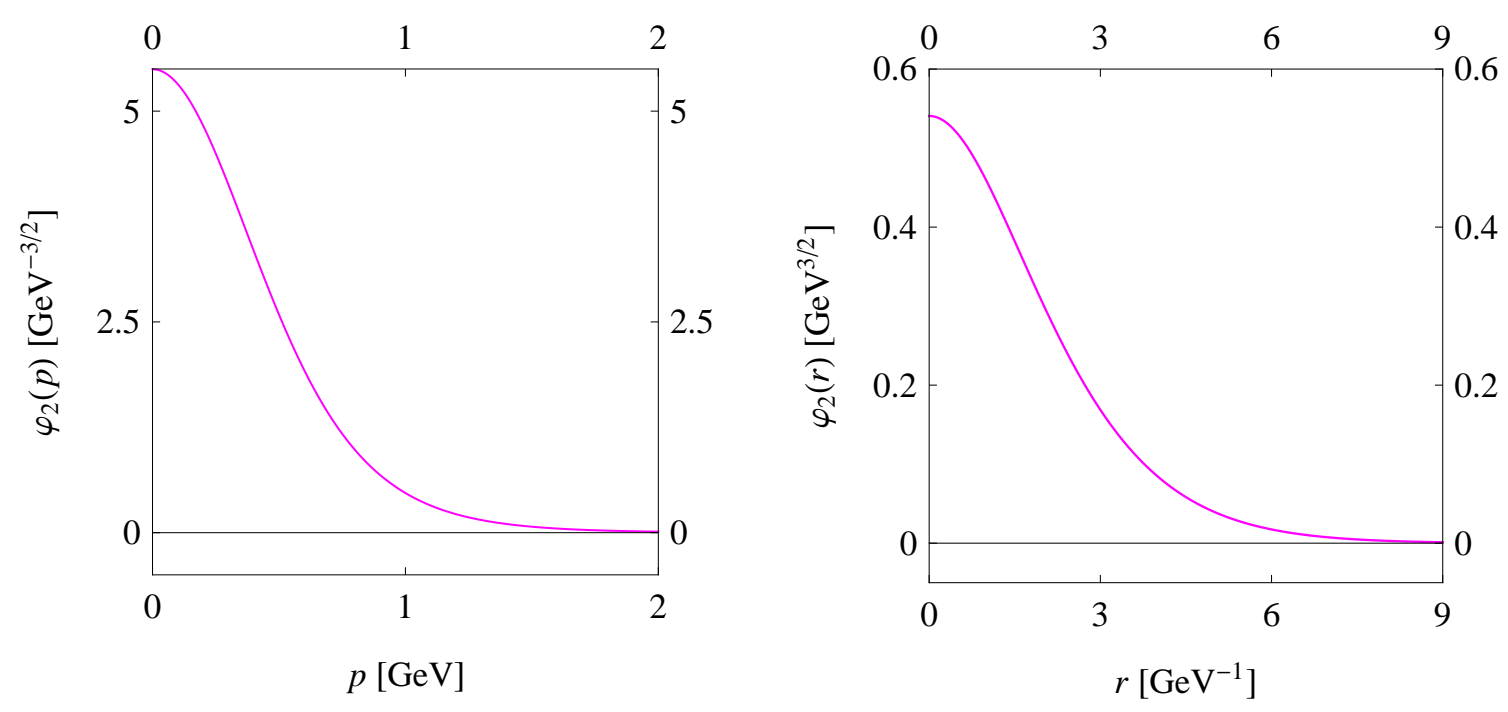

Figure 2: Nonzero Salpeter component in momentum $\left[\varphi_{2}(p)\right.$, left] and configuration $\left[\varphi_{2}(r)\right.$, right $]$ space [8].

Our bound-state equation in configuration space then yields the potential $V(r)$. This potential's unexpected square-well shape resemblance we consider as this exercise's true quintessence (Fig. 3).

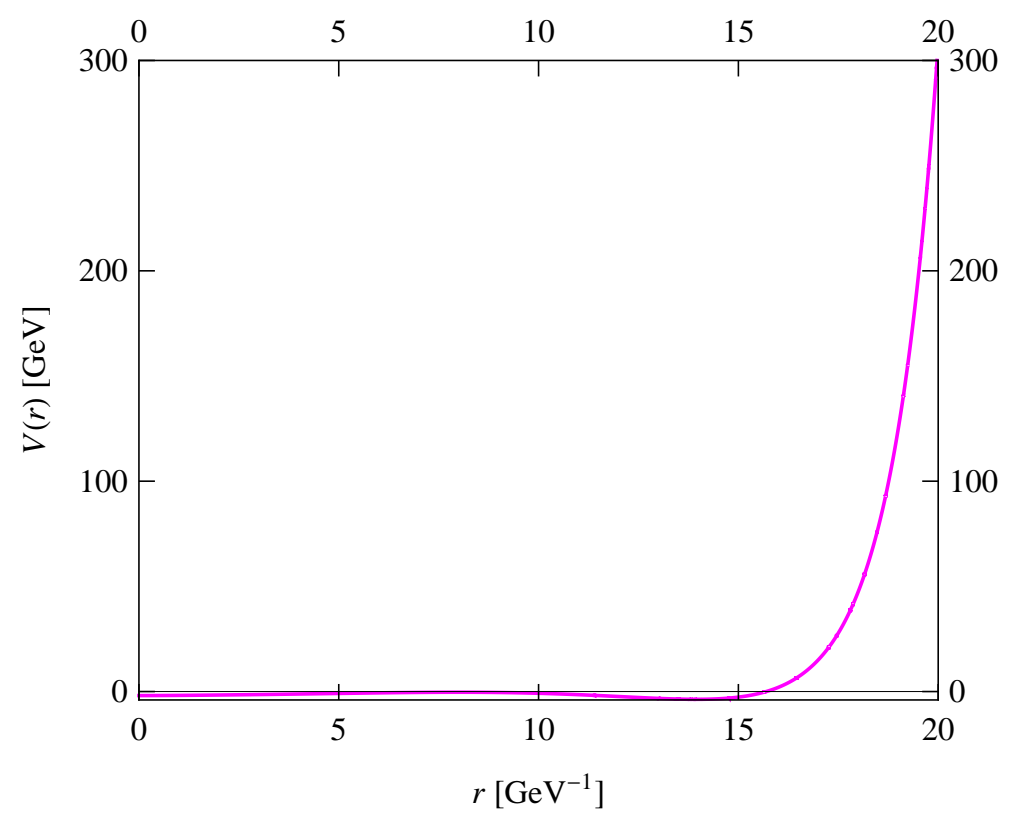

Figure 3: Configuration-space central potential $V(r)$ [8], arising from a chiral-limit quark propagator [13,14] as input by inverting our three-dimensional bound-state equation [3] with Fierz-symmetric interaction kernel.

Not surprisingly, a brief scrutiny with the naked eye reveals [8] that the ground-state solution of our three-dimensional bound-state equation with potential $V(r)$ fixed by inversion inevitably entails a reasonable size of the pion: its average interquark distance, $\langle r\rangle=0.483 \mathrm{fm}$, and root-mean-square radius, $\sqrt{\left\langle r^{2}\right\rangle}=0.535 \mathrm{fm}$, predicted by the starting point $\varphi_{2}(r)$ of our inversion approach match the experimentally determined value of its electromagnetic charge radius, $\sqrt{\left\langle r_{\pi}^{2}\right\rangle}=(0.672 \pm 0.008) \mathrm{fm}$. 
Consistency of our inversion results can be established by numerically solving, for the effective interaction potential derived thereby, the bound-state equation either variationally (Fig. 4, left) or by expansion over a suitable basis of function space (Fig. 4, right). Our approach passes, of course, this test with flying colours. In both cases, the overlap of wave-function input and outcome equals unity.
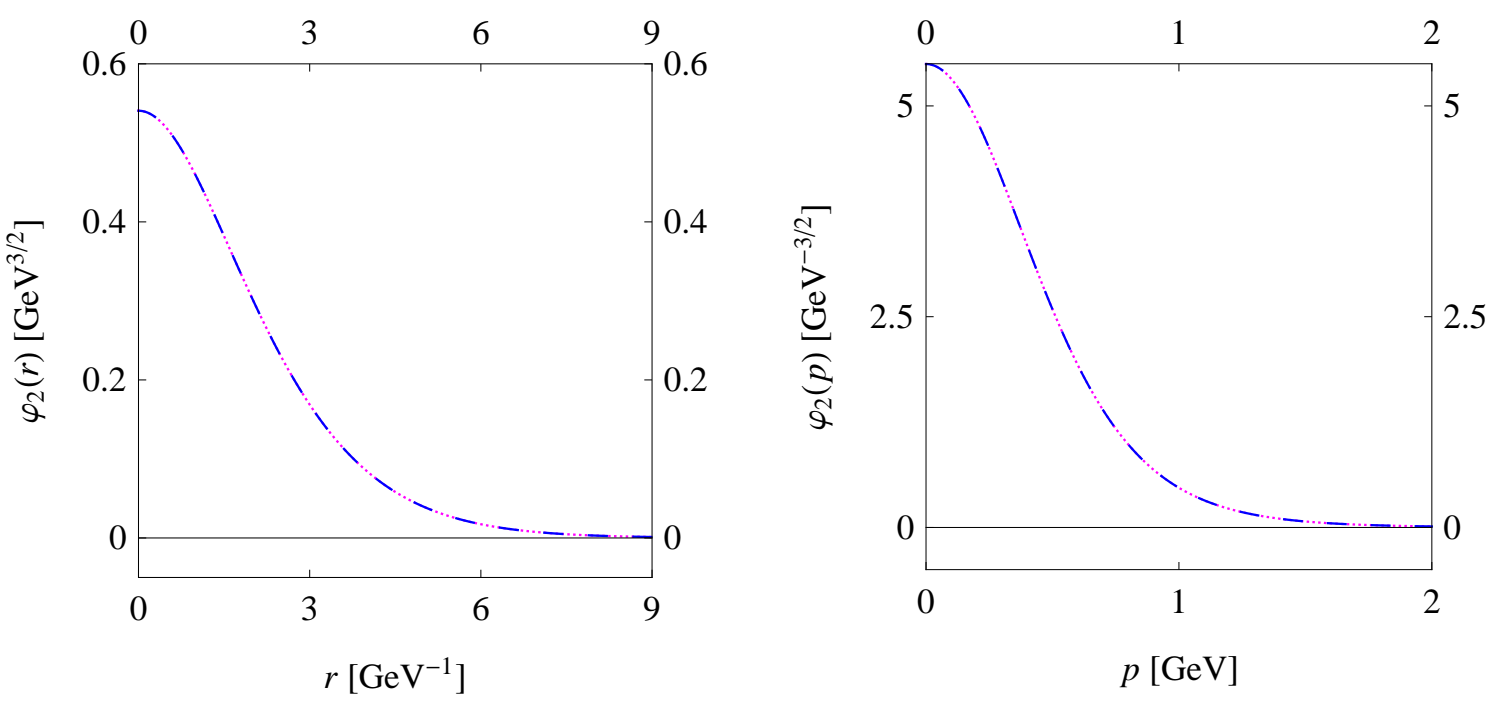

Figure 4: Actual ground-state solutions (dotted) to our three-dimensional bound-state equation [3], obtained straightforwardly by application of variational techniques $\left[\varphi_{2}(r)\right.$, left] or a conversion to an equivalent matrix eigenvalue problem $\left[\varphi_{2}(p)\right.$, right], vs. the initial Salpeter component (dashed), this inversion's starting point.

\section{References}

[1] W. Lucha and F. F. Schöberl, Phys. Rev. D 87 (2013) 016009, arXiv:1211.4716 [hep-ph].

[2] W. Lucha, Proc. Sci., EPS-HEP 2013 (2013) 007, arXiv:1308.3130 [hep-ph].

[3] W. Lucha and F. F. Schöberl, J. Phys. G 31 (2005) 1133, arXiv:hep-th/0507281.

[4] Z.-F. Li, W. Lucha, and F. F. Schöberl, Mod. Phys. Lett. A 21 (2006) 1657, arXiv:hep-ph/0510372.

[5] W. Lucha and F. F. Schöberl, Phys. Rev. D 92 (2015) 076005, arXiv:1508.02951 [hep-ph].

[6] W. Lucha and F. F. Schöberl, Phys. Rev. D 93 (2016) 056006, arXiv:1602.02356 [hep-ph].

[7] W. Lucha and F. F. Schöberl, Phys. Rev. D 93 (2016) 096005, arXiv:1603.08745 [hep-ph].

[8] W. Lucha and F. F. Schöberl, Int. J. Mod. Phys. A 31 (2016) 1650202, arXiv:1606.04781 [hep-ph].

[9] W. Lucha, EPJ Web Conf. 129 (2016) 00047, arXiv:1607.02426 [hep-ph].

[10] W. Lucha, EPJ Web Conf. 137 (2017) 13009, arXiv:1609.01474 [hep-ph].

[11] E. E. Salpeter, Phys. Rev. 87 (1952) 328.

[12] P. Maris, C. D. Roberts, and P. C. Tandy, Phys. Lett. B 420 (1998) 267, arXiv:nucl-th/9707003.

[13] P. Maris and P. C. Tandy, Phys. Rev. C 60 (1999) 055214, arXiv:nucl-th/9905056.

[14] P. Maris, in Proc. Int. Conf. on Quark Confinement and the Hadron Spectrum IV, eds. W. Lucha and K. Maung Maung (World Scientific, Singapore, 2002), p. 163, arXiv:nucl-th/0009064. 